\title{
Flow Around Islands in Rupert Bay: An Investigation of the Bottom Friction Effect
}

\author{
R. GRANT INGRAM ${ }^{1}$ \\ Institute of Oceanography, McGill University, Montreal, Quebec, Canada \\ VINCENT H. CHU \\ Department of Civil Engineering, McGill University, Montreal, Quebec, Canada
}

\begin{abstract}
Aerial photographs of transverse shear flows around islands in Rupert Bay, a shallow and turbid estuary in northern Quebec, were studied for the effect of bottom friction. The island wake depends on the stability of the transverse shear layers developed along the two sides of the island. Two basic patterns of wake flows were observed. A vortex street wake was formed when transverse shear was large, as in flows around small islands. On the other hand, for small transverse shear, as in flows around large islands, the shear layers were stabilized by bottom friction and, in that case, a clear water wake of low turbulence and low sediment concentration was observed. Laboratory demonstration of the bottom friction influence on the turbulent wake in shallow water was made using a shallow water table. A similarity solution was obtained for the wake flow in the far-field region, and numerical calculations were made of the recirculating flow in the wake bubble. A wake stability parameter was introduced for wake classification and for correlation of field and laboratory data. In Rupert Bay, vortex street wakes were observed in nine out of 27 events when the wake stability parameter was less than a critical value. This result is consistent with the laboratory and numerical simulations.
\end{abstract}

\section{INTRODUCTION}

Rupert Bay is a shallow, turbid river estuary located off the southeast corner of James Bay (northern Canada), approximately $60 \mathrm{~km}$ long and $20 \mathrm{~km}$ wide. It receives a mean annual freshwater input of $2340 \mathrm{~m}^{3} / \mathrm{s}$ from four rivers: the Nottaway $\left(1020 \mathrm{~m}^{3} / \mathrm{s}\right)$, Broadback $\left(320 \mathrm{~m}^{3} / \mathrm{s}\right)$, Rupert $\left(870 \mathrm{~m}^{3} / \mathrm{s}\right)$, and a small river, the Pontax $\left(130 \mathrm{~m}^{3} / \mathrm{s}\right)$. The general orientation of the bay is in a NW-SE direction.

The presence of a large number of islands and turbid waters make Rupert Bay ideal for flow visualization in a natural setting (Figure 1). Vortex motions, with a horizontal length scale hundreds of times larger than the depth of water, are observed occasionally as the flow passes around the islands. The estuary is quite shallow, with depths (low water) of 3 to 5 $\mathrm{m}$ characterizing most of the region (Figure 2). Downstream of Stag Rock, three deeper channels extending inland from James Bay can be found. Maximum channel depths are approximately $15 \mathrm{~m}$.

Tides in Rupert Bay are comparable in magnitude to the depth, with a mean semidiurnal range at Stag Island of $2 \mathrm{~m}$ and spring tides of up to $3 \mathrm{~m}$. Average tidal current speeds in the central bay area range from 50 to $100 \mathrm{~cm} / \mathrm{s}$, which are an order magnitude greater than the mean flow $(5-10 \mathrm{~cm} / \mathrm{s})$.

During the ice free period, high turbidity prevails in most sections of the bay. Patterns in the flow field are often shown by the turbidity difference between masses of water from different sources. Mixing in the transverse direction is limited in length scale, possibly by the stable influence of bottom friction.

\footnotetext{
'Now at Meteorology Department, McGill University, Montreal, Quebec, Canada.

Copyright 1987 by the American Geophysical Union.

Paper number $7 \mathrm{C} 0679$.

0148-0227/87/007C-0679\$05.00
}

Two basic patterns of wake flow around the islands are observed, depending on the size of the island: (1) a turbulent wake bubble trailed by a vortex street wake as shown in Figures $3 a-3 c$ or (2) a clear water wake bubble of low turbulence and low sediment concentration as in Figures $3 d-3 f$. The aerial photographs shown in Figure 3 were taken by the Canada Centre for Remote Sensing from an altitude of $3050 \mathrm{~m}$ using a Wild RC10 Camera, directed down from the underside of the aircraft. Oceanographic field studies were under way in Rupert Bay at the time of overflights and were used to provide sediment, current, and salinity information as ground truth in some of the areas.

The turbulent motions in the bay consist of two distinct length scales. The large-scale vortex motions are created by the transverse shear. The small-scale motions, created by bottom shear and limited by water depth, are not visible because of their small length scale when compared to the resolution of the photographs. From a depth-averaged point of view the transverse shear flow is laminar if the scale of turbulent motion is limited by the depth of flow. The flow becomes turbulent as the transverse shear flow becomes unstable.

Although the Reynolds numbers are very large (typically $10^{7}-10^{10}$, based on cross-stream diameter), the flow around the islands is remarkably similar in appearance to the twodimensional wake flow observed in the laboratory at low Reynolds number (see, e.g., Batchelor [1967, Figure 4.12.6]).

The low Reynolds number flow in the laboratory is strongly influenced by molecular viscosity: The stability of the wake bubble depends on the stability of the shear layers along the two sides of the wake bubble. The shear layer is stable at a low Reynolds number of about 10 . As the Reynolds number increases, the shear layers become less stable, and oscillation begins. The interaction of the two unstable shear layers is the cause for the formation of the well-known Karman vortex street. At still higher Reynolds numbers, vortex motion begins 


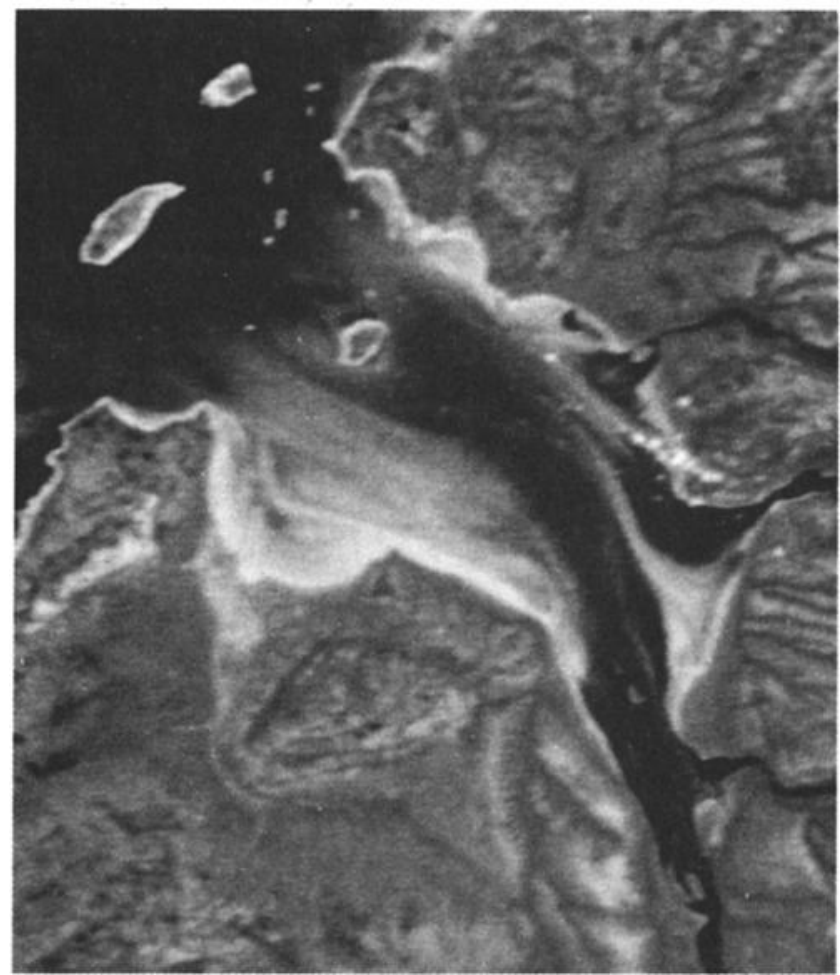

Fig. 1. Landsat photograph of Rupert Bay (James Bay, Canada). Water masses from the four rivers of different sediment concentrations remain distinct over a major portion of the bay.

to cascade its energy toward smaller scale, and a more turbulent wake emerges.

The island wakes in Rupert Bay are definitely not affected by molecular viscosity, but are subject to the stabilizing influence of bottom friction. In an analysis of laminar and parallel flows in shallow depths, Chu et al. [1983] introduced a bottom friction stability parameter

$$
S=c_{f} \bar{U} / 2 h \bar{U}_{y}
$$

in which $c_{f}$ is a bottom friction coefficient, $h$ is the water depth, $\bar{U}$ and $\bar{U}_{y}$ are the velocity and velocity gradient at the inflexion point, and $y$ is the coordinate in the transverse direction. This bottom friction stability parameter is a relative measure of the stabilizing effect of bottom friction and the destabilizing effect of the transverse shear. Chu et al. [1983] obtained critical values for stability of transverse shear flows: Transverse shear flow is stable if the stability parameter $S$ exceeds 0.120 and 0.145 , for hyperbolic tangent and hyperbolic secant velocity profiles, respectively.

The flow around an island in Rupert Bay is more complex than parallel flow. However, the bottom friction parameter is still a useful indicator for the relative influence of bottom friction and transverse shear. In general, the flow around the larger islands is more stable because the magnitude of the transverse shear, $\bar{U}_{y}$, being inversely proportional to the horizontal length scale of the shear flow, is smaller. When both the length and velocity scales are correctly chosen, a wake stability parameter can be introduced for the classification of flows around islands in a shallow body of water.

The flow around an island has been of considerable interest for some time. Similar flow patterns have been observed in the Earth's atmosphere and in various coastal waters. Earlier work was primarily concerned with the similarity of these flows with laboratory observations of two-dimensional shear

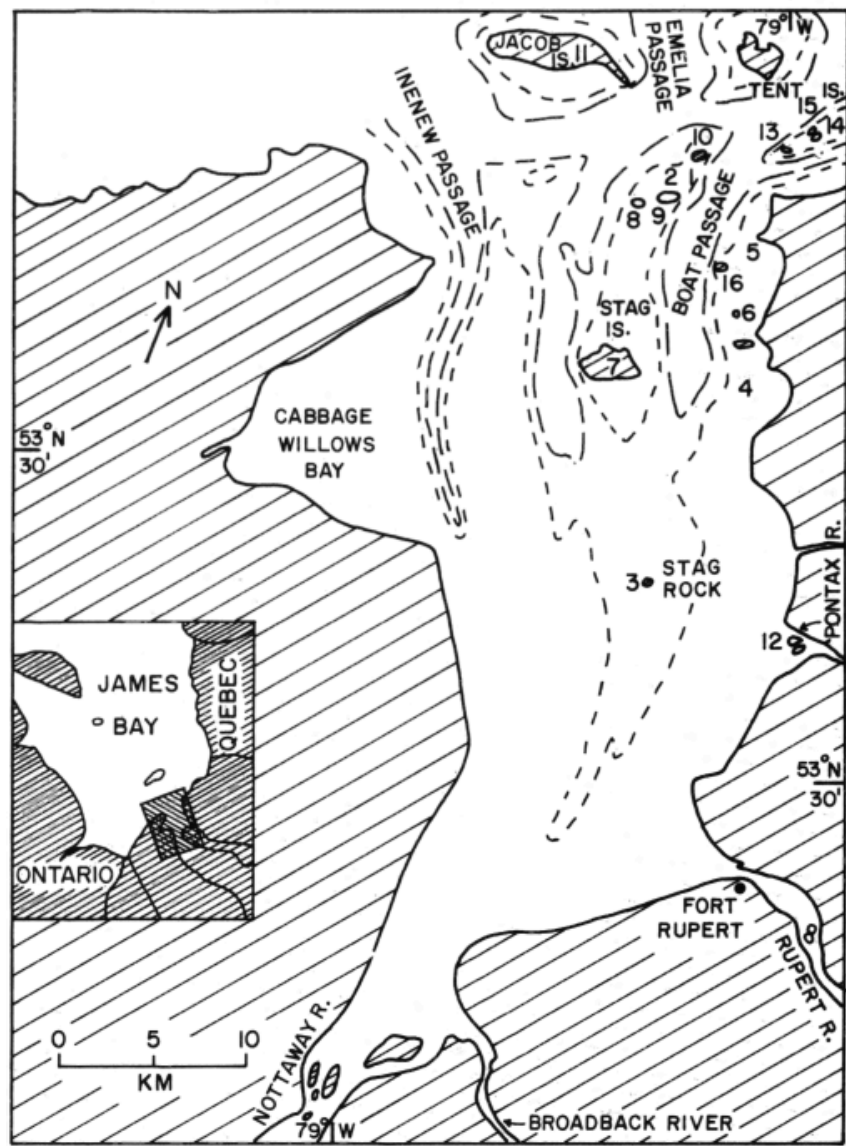

Fig. 2. Bathymetry of Rupert Bay in meters. The island locations are identified by numbers $N$, which are used in the rest of the paper and in Table 1; the short dashes denote the 3-m depth contour, and the long dashes denote the 6-m depth contour.

flow [e.g., Chopra and Hubert, 1965; Burger and Wille, 1972]. But recent studies have recognized the distinctive nature of these natural shear flows from the conventional laboratory problem. Perhaps the most relevant study was that of Wolanski et al. $[1984 a, b]$, who introduced a parameter $P$ to account for the friction influence of the bottom boundary layer inside the island wake bubble, defined as follows:

$$
P=U_{1} h^{2} / v_{T} D
$$

where $h$ is water depth, $U_{1}$ is the free stream velocity, $D$ the cross-stream diameter of the island, and $v_{T}=0.15 h U_{*}$ is an eddy viscosity related to the bottom friction velocity. They suggested that a critical value $\boldsymbol{P}_{c}$, of order of unity, should exist and that a vortex street wake is not possible for $P \ll P_{c}$.

The principal objective of this paper is to present a collection of aerial photographs of the flows around islands in Rupert Bay and to show how the length scale of turbulent motion in the bay is limited by the stabilizing influence of bottom friction. To this end, the flows around small and large islands are described analytically and compared with both laboratory and field observations.

The study begins with an analytical description of the vortex street wake. It is shown how the vortex street wake may be formed behind small islands and how it is "nullified" eventually in the far-field region by the presence of bottom friction. The analytical solution of the vortex street wake is compared with laboratory observations. Similarity parameters are introduced from the analytical description which are ap- 


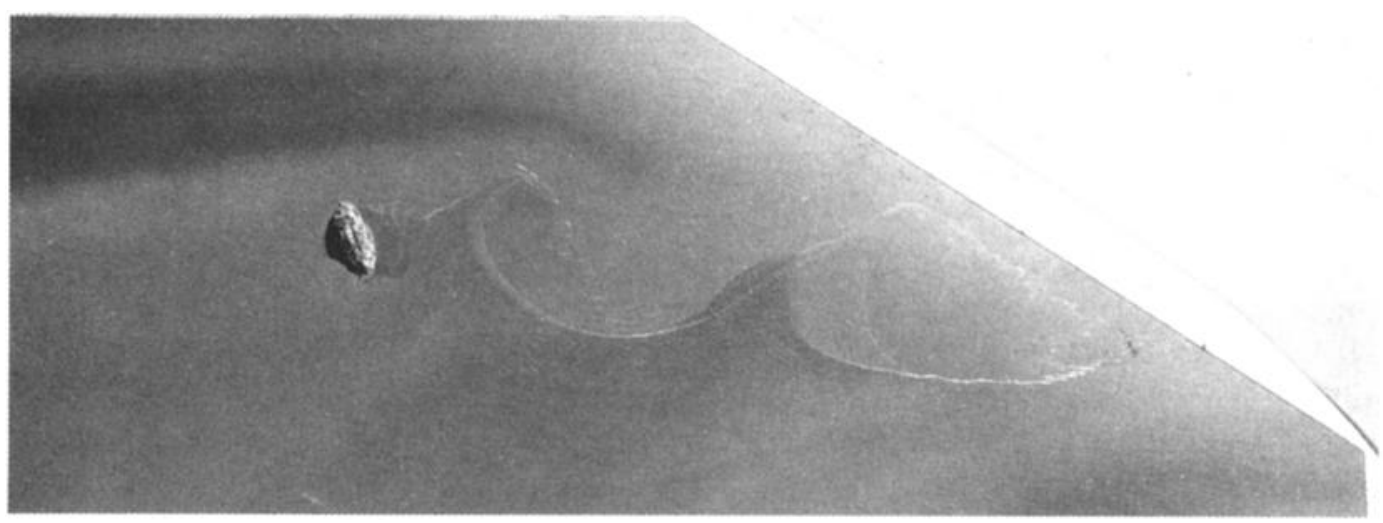

Fig. 3a. Vortex street wake, island diameter: $160 \mathrm{~m}$.

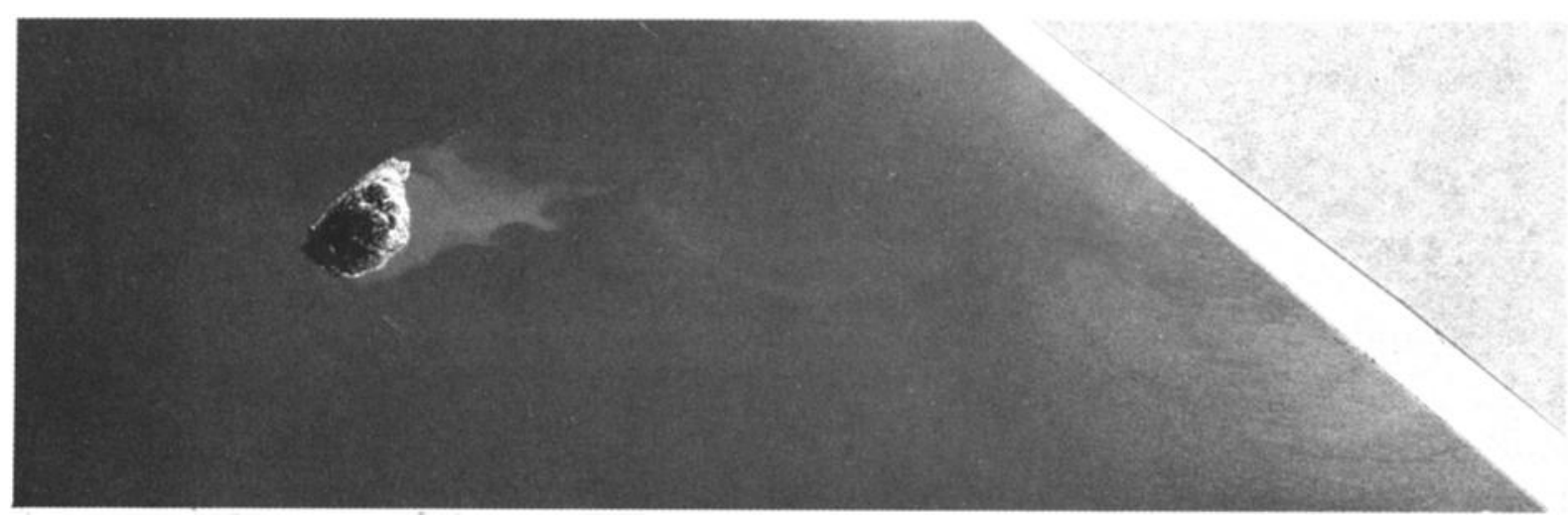

Fig. $3 b$. Vortex street wake, island diameter: $280 \mathrm{~m}$.

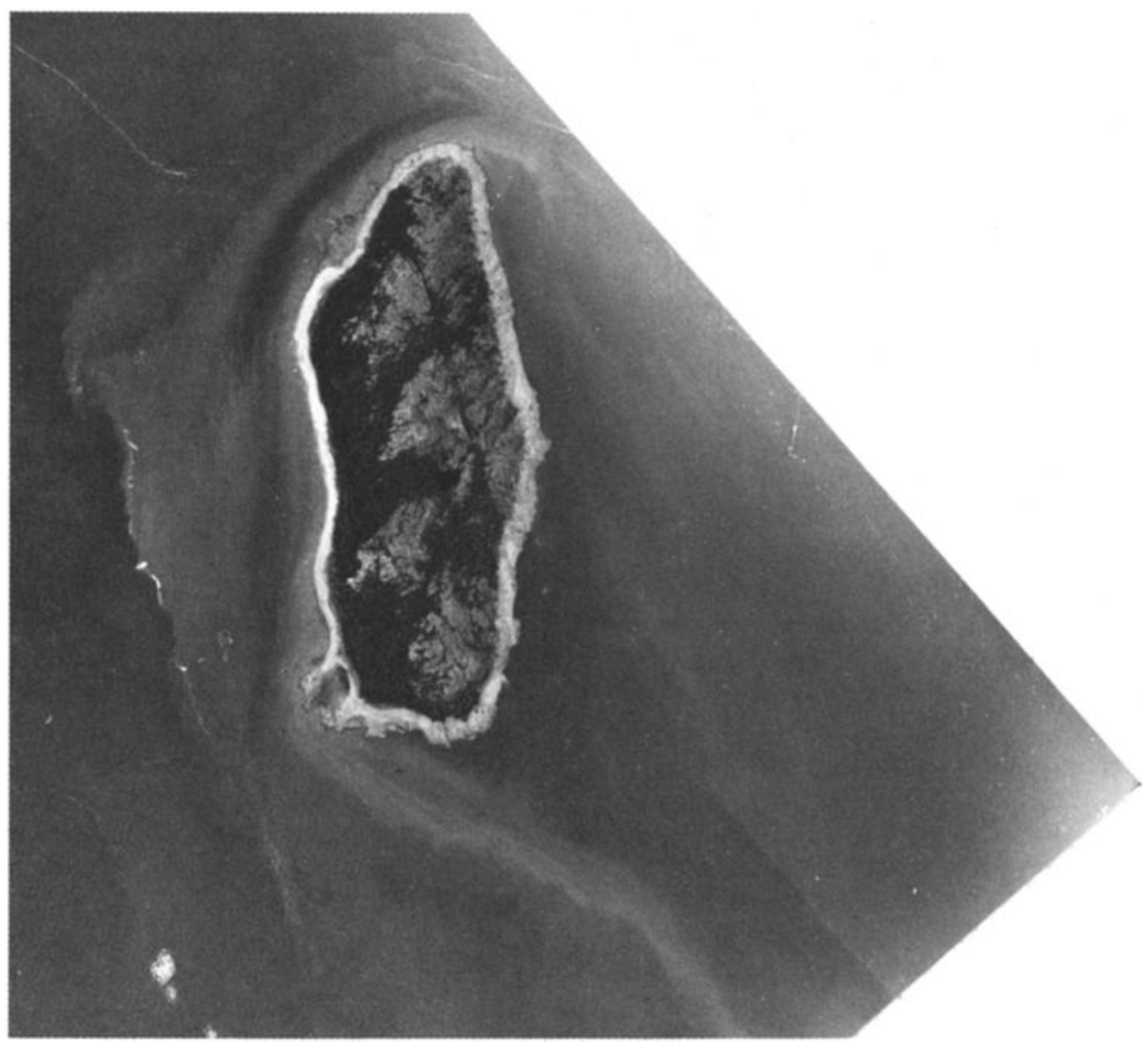

Fig. 3c. Vortex street wake and clear water wakes near low tide.

Fig. 3. Examples of vortex street wake and clear water wakes. All photos have same scale. 


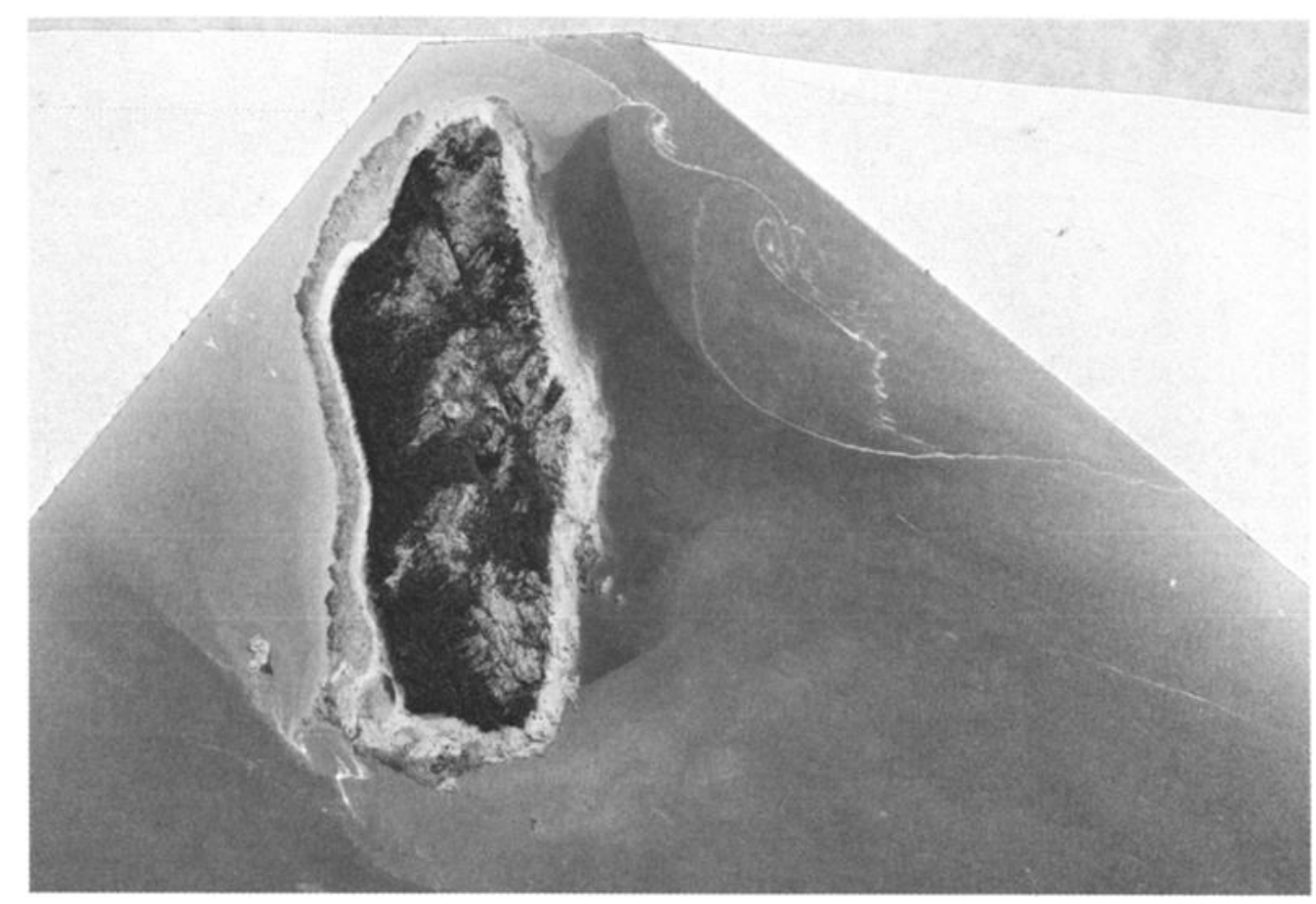

Fig. 3d. Clear water wakes only in same area as Figure $3 c$ near high tide.

plied to the data obtained from the field observations. Results of a numerical simulation of flow around an obstacle in shallow water are also given to demonstrate how a clear water wake may be formed behind a large island by the "sheltering" effect.

\section{Analytical Description of VorteX Street Wakes in Shallow Waters}

\subsection{Shallow Water Wake Equation}

The bottom friction influence on the vortex street wake (i.e., on the flow behind small islands) can be readily determined from a depth-averaged formulation. By depth averaging, the small-scale turbulent motion is filtered out (as it is invisible in the aerial photographs); its effect on the depth-averaged flow field is replaced by a bottom friction term in the equation.

Figure 4 shows a vortex street wake and the associated depth-averaged velocity profile. The velocity outside the wake is $U_{1}$, and the velocity defect in the wake is $u$. The equation for the wake, obtained from depth averaging with a rigid lid approximation, is

$$
-U_{1} \frac{\partial u}{\partial x}=-\frac{\partial P}{\partial x}-\frac{c_{f}}{2 h}\left(U_{1}-u\right)^{2}-\frac{\partial}{\partial y}\left[v_{T} \frac{\partial u}{\partial y}\right]
$$

in which $\partial P / \partial x$ is the longitudinal pressure gradient under the rigid lid, $c_{f}$ the bottom friction coefficient, $h$ the water depth, and $v_{T}$ the eddy viscosity. For flow outside the wake, $u \rightarrow 0,(1)$ becomes

$$
0=-\frac{\partial P}{\partial x}-\frac{c_{f}}{2 h} U_{1}^{2}
$$

Eliminating the pressure gradient term from (1) and (2) gives

$$
U_{1} \frac{\partial u}{\partial x}=-\left(\frac{c_{f} U_{1}}{h}\right) u+\frac{\partial}{\partial y}\left[v_{T} \frac{\partial u}{\partial y}\right]
$$

which is the turbulent wake equation with a bottom friction term (or the diffusion equation with frictional damping). In deriving (3) the far-wake approximation was used, i.e., $u \ll U_{1}$ and $\partial / \partial x \ll \partial / \partial y$.

\subsection{Integral Constraint and Similarity Solution}

Integration of (3) across the wake gives

$$
\frac{d}{d x}\left[U_{1} \int_{-\infty}^{+\infty} u d y\right]=-\left(\frac{c_{f}}{h}\right) U_{1} \int_{-\infty}^{+\infty} u d y
$$

Integration with respect to $x$ leads to an expression for the momentum defect,

$$
M=U_{1} \int_{-\infty}^{+\infty} u d y=M_{0} \exp \left[-\frac{c_{f}}{h} x\right]
$$

$M$ decays exponentially because of bottom friction. The initial momentum defect is the drag on the island, i.e., $M_{0}=\frac{1}{2} C_{D}$ $D U_{1}{ }^{2}$, in which $C_{D}$ is a drag coefficient and $D$ is the crossstream diameter of the island. The wake becomes nullified as the momentum defect is reduced to zero in the far-field region (see Chu and Abdelwahed [1981] for the concept of wake nullification).

Despite the presence of the bottom friction term a similarity solution for the shallow wake is possible. If the eddy viscosity is assumed to take the form

$$
v_{T}(x)=U_{1}(d \delta / d x) \delta /(2 \ln 2)
$$

the similarity solution would be the Gaussian profile

$$
u / u_{m}=\exp \left[-(\ln 2)\left(y^{2} / \delta^{2}\right)\right]
$$

in which $u_{m}(x)$ is the maximum velocity defect along the centerline and $\delta$ is the half width of the wake, defined such that $u=u_{m} / 2$ at $y= \pm \delta$.

The ratio of the two velocity scales, $u_{m}$ and $U_{1}(d \delta / d x)$, from 


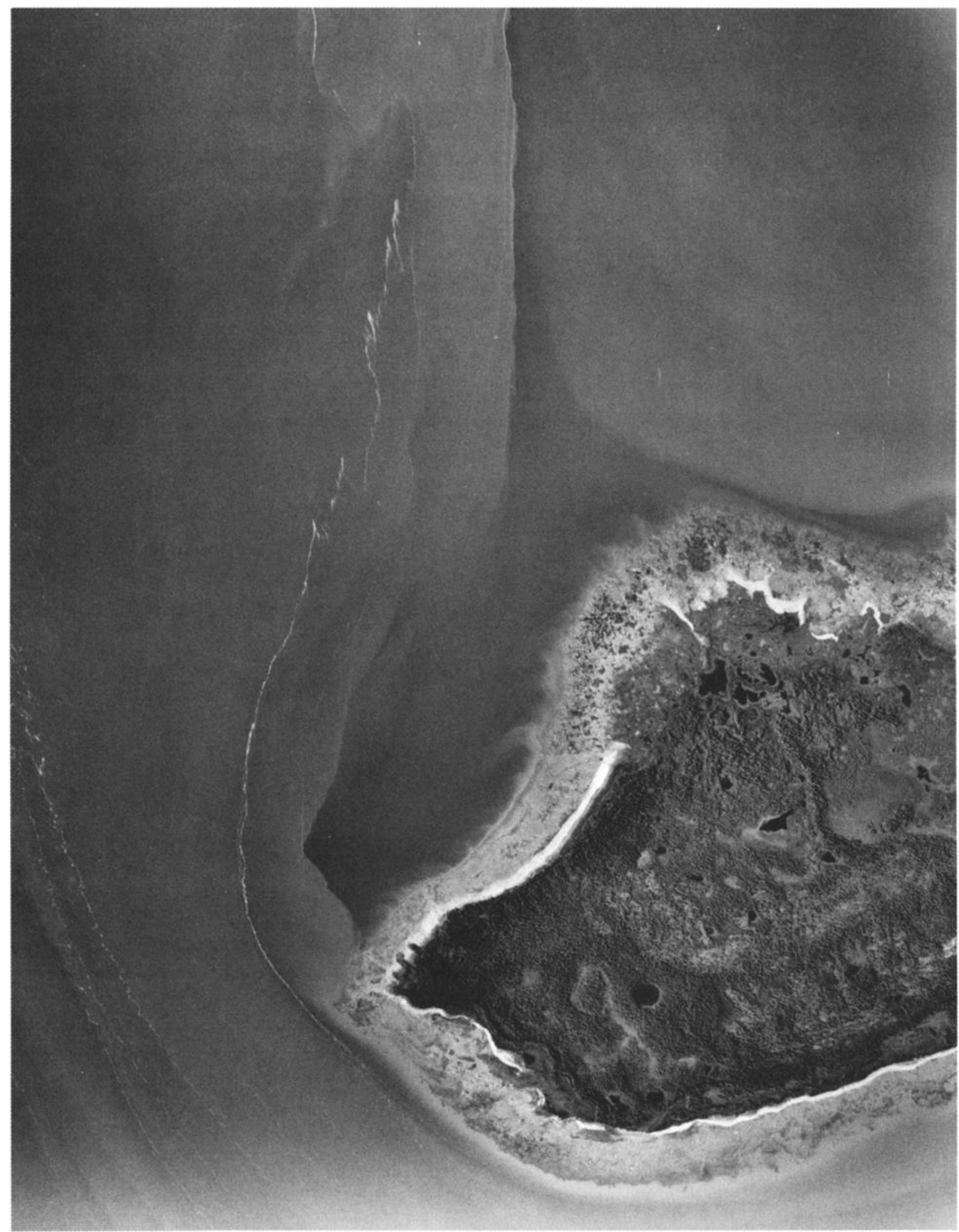




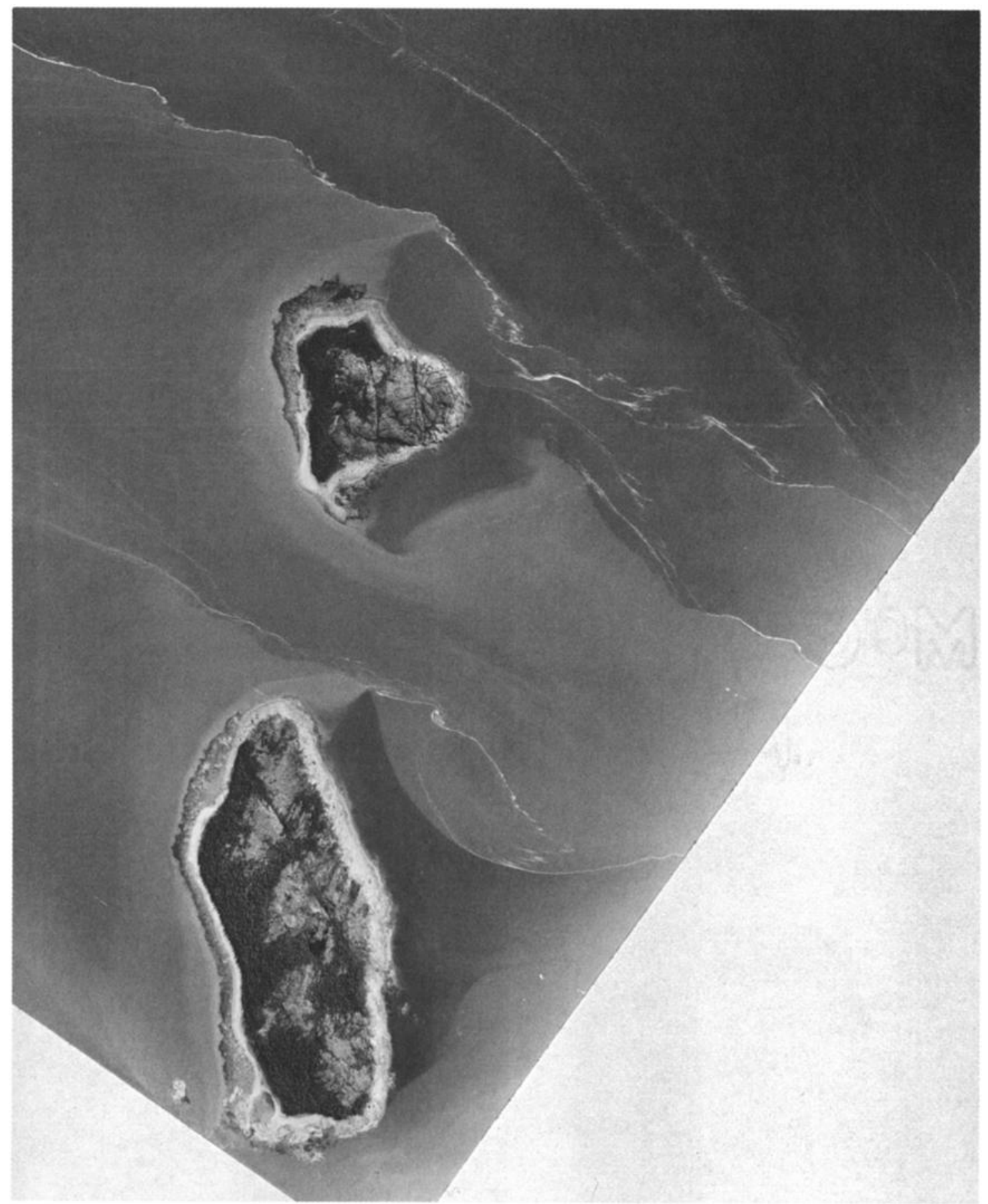

Fig. $3 f$ Clear water wake, island diameters: $560,1310 \mathrm{~m}$.

the similarity solution defines an entrainment coefficient $\alpha^{*}$ as follows:

$$
U_{1}(d \delta / d x)=\alpha^{*} u_{m}
$$

The overall turbulent activity in the wake depends on the magnitude of either the eddy viscosity $v_{T}$ or the entrainment coefficient $\alpha^{*}$, which are related through (6) and (8).

Solutions for $u_{m}(x)$ and $\delta(x)$ are obtained using the entrainment hypothesis, equation (8), and the integral constraint, equation (5). For a Gaussian velocity profile, the momentum defect is given by

$$
\begin{aligned}
& M=U_{1} u_{m} \delta \int_{-\infty}^{+\infty} \exp \left[-(\ln 2) \eta^{2}\right] d \eta \\
& \quad=2.13 U_{1} u_{m} \delta=\frac{1}{2} C_{D} U_{1}^{2} \exp \left[-\frac{c_{f} x}{h}\right]
\end{aligned}
$$

Multiplying (8) by $2 \delta U_{1}$ and making use of (9),

$$
U_{1}^{2} \frac{d \delta^{2}}{d x}=2 \alpha^{*} U_{1} u_{m} \delta=\frac{\alpha^{*}}{2.13} C_{D} U_{1}^{2} \exp \left[-\frac{c_{f} x}{h}\right]
$$




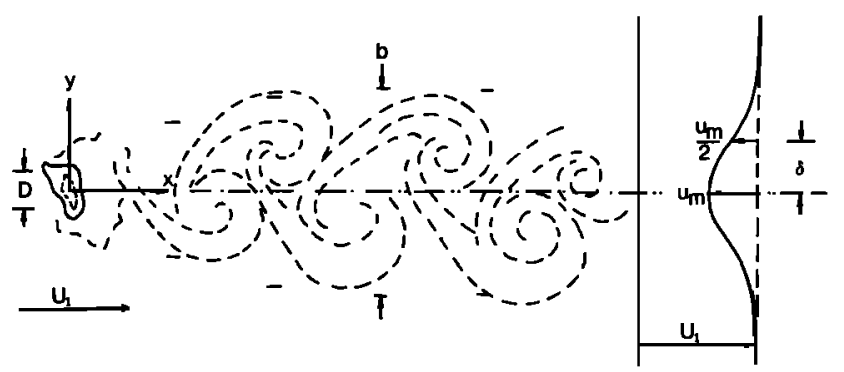

Fig. 4. Definition sketch of wake in shallow depth. Dashed line shows the cloud pattern downstream of the island of Madeira as outlined by Burger and Wille [1972].

Integrating this equation with respect to $x$ gives

$$
\delta^{2}=\int_{0}^{x} \alpha C_{D} \exp \left[-\frac{c_{f} x}{h}\right] d x
$$

in which $\alpha=\alpha^{*} / 2.13$ is an alternative expression for the entrainment coefficient. Equation (11) can be formulated in terms of dimensionless variables such that

$$
\tilde{\delta}^{2}=C_{D} \int_{0}^{x} \alpha \exp [-\tilde{x}] d \tilde{x}
$$

where

$$
\tilde{\delta}=c_{f} \delta / h \quad \tilde{x}=c_{f} x / h \quad \tilde{U}_{1}=U_{1} / u_{m}
$$

\subsection{Entrainment Coefficient}

As one approaches deep water $\left(c_{f} / h \rightarrow 0\right)$, the entrainment coefficient becomes constant, and (11) reduces to

$$
\delta^{2}=\alpha_{0} C_{D} x
$$

The entrainment coefficient for this limiting case, $\alpha_{0}$, is determined by comparing (14) with the expression $\delta^{2}=0.09 x$, obtained from fitting the experimental data of a wake behind a circular cylinder [Townsend, 1956]; thus $\alpha_{0}=0.09$ if $C_{D}=1.0$.

For shallow water the entrainment process is affected by the stabilizing influence of bottom friction stresses. In general, the entrainment coefficient $\alpha$ is not a constant, but is dependent on the local bottom friction parameter

$$
S=c_{f} \bar{U} / 2 h \bar{U}
$$

in which $\bar{U}$ and $\bar{U}_{y}$ are the velocity and velocity gradient at the inflexion point. In an experimental study of a turbulent mixing layer in open channel flow, Babarutsi and Chu [1985] proposed the following expression for the entrainment coefficient:

$$
\begin{array}{ll}
\alpha=\alpha_{0}\left[1-\left(S / S_{c}\right)\right] & S \leq S_{c} \\
\alpha=0 & S>S_{c}
\end{array}
$$

Here the entrainment coefficient decreases as the bottom friction parameter increases, becoming zero once $S$ exceeds the critical value of $S_{c}$. For the turbulent mixing layer the critical value $S_{c}$ was approximately 0.085 . The value for a turbulent wake is unknown, but can be estimated from stability considerations, as in Chu et al. [1983]. Calculations shown in the appendix give a critical value of $S_{c} \simeq 0.16 \sim 0.17$.

At the inflexion point of the Gaussian velocity profile, $\bar{u}=0.61 u_{m}, \bar{u}_{y}=0.71 u_{m} / \delta$, and $\bar{U}=U_{1}-u=U_{1}-0.61 u_{m}$.
Making use of these relationships, we can express the bottom friction parameter in terms of dimensionless variables $\tilde{\delta}$ and $\tilde{U}_{1}$ as follows:

$$
S=0.700 \delta\left[\tilde{U}_{1}-0.6065\right]
$$

in which

$$
\tilde{U}_{1}=\frac{2(2.13)}{C_{D}} \frac{\tilde{\delta}}{\tilde{D}} \exp [+\tilde{x}]
$$

according to (9). Equation (13), together with the expressions for the entrainment coefficient, given by (14), (17), and (18), forms a complete set of relationships, which is used to determine the growth of the wake, i.e., the relation $\tilde{\delta}=\tilde{\delta}(\tilde{x})$, uniquely.

The development of an island wake in shallow water is quite different from the two-dimensional wake in deep water. The width of the two-dimensional wake in deep water increases with distance from the obstacle without bound (equation (14)). In shallow water the extent of the wake is limited by the influence of bottom friction. The wake width approaches an asymptote as the bottom friction parameter $S$ reaches the critical value of $S_{c}$. The momentum defect in the wake continues to decay exponentially after the asymptotic width is attained.

The extent of the wake depends critically on the entrainment hypothesis. A significantly larger asymptote would be predicted by (13) if the entrainment coefficient were assumed to be a constant instead of dependent on the bottom friction parameter (through (16)).

In the laboratory and field investigations presented in this paper, the wake width is obtained from flow visualization. The location and the extent of the asymptote are used to evaluate the bottom friction influence on the wake.

\section{Flow Visualization ON A WATER TABLE}

Flow visualization experiments were carried out on a shallow water table in the laboratory. The water table was $196 \mathrm{~cm}$ long and $70.5 \mathrm{~cm}$ wide and was illuminated from below by fluorescent light. A flat plate was placed normal to the shallow open channel flows. The wake was made visible by injection of dye upstream of the flat plate. Six tests were conducted. Table 1 summarizes the test conditions.

The friction coefficients for the open channel flow were calculated according to the suggestion by Chow [1959], i.e., use the smooth pipe flow formula but replace the pipe diameter by 4 times the hydraulic radius (e.g., $\operatorname{Re}=4 h U_{1} / v$ ). The drag coefficient of the flat plate, $C_{D}$, is assumed to have a value of 1.1 (for a body with a small length to diameter ratio or small $h / D)$.

TABLE 1. Test Conditions

\begin{tabular}{lcccccc}
\hline Text & $\begin{array}{c}D, \\
\mathrm{~cm}\end{array}$ & $\begin{array}{c}h, \\
\mathrm{~cm}\end{array}$ & $\begin{array}{c}U_{1}, \\
\mathrm{~cm} / \mathrm{s}\end{array}$ & $c_{\boldsymbol{f}}$ & $\begin{array}{c}R e= \\
4 h U_{1} / \nu\end{array}$ & $\begin{array}{c}S_{w}= \\
c_{\boldsymbol{f}} D / h\end{array}$ \\
\hline A1 & 6.0 & 1.4 & 25.4 & 0.07 & 11,000 & 0.031 \\
$\mathrm{~A} 2$ & 5.0 & 1.4 & 25.4 & 0.07 & 11,000 & 0.026 \\
$\mathrm{A3}$ & 2.5 & 1.4 & 25.4 & 0.07 & 11,000 & 0.013 \\
B1 & 6.0 & 0.9 & 16.9 & 0.10 & 4,700 & 0.065 \\
B2 & 5.0 & 0.9 & 16.9 & 0.10 & 4,700 & 0.054 \\
B3 & 2.5 & 0.9 & 16.9 & 0.10 & 4,700 & 0.027 \\
\hline
\end{tabular}



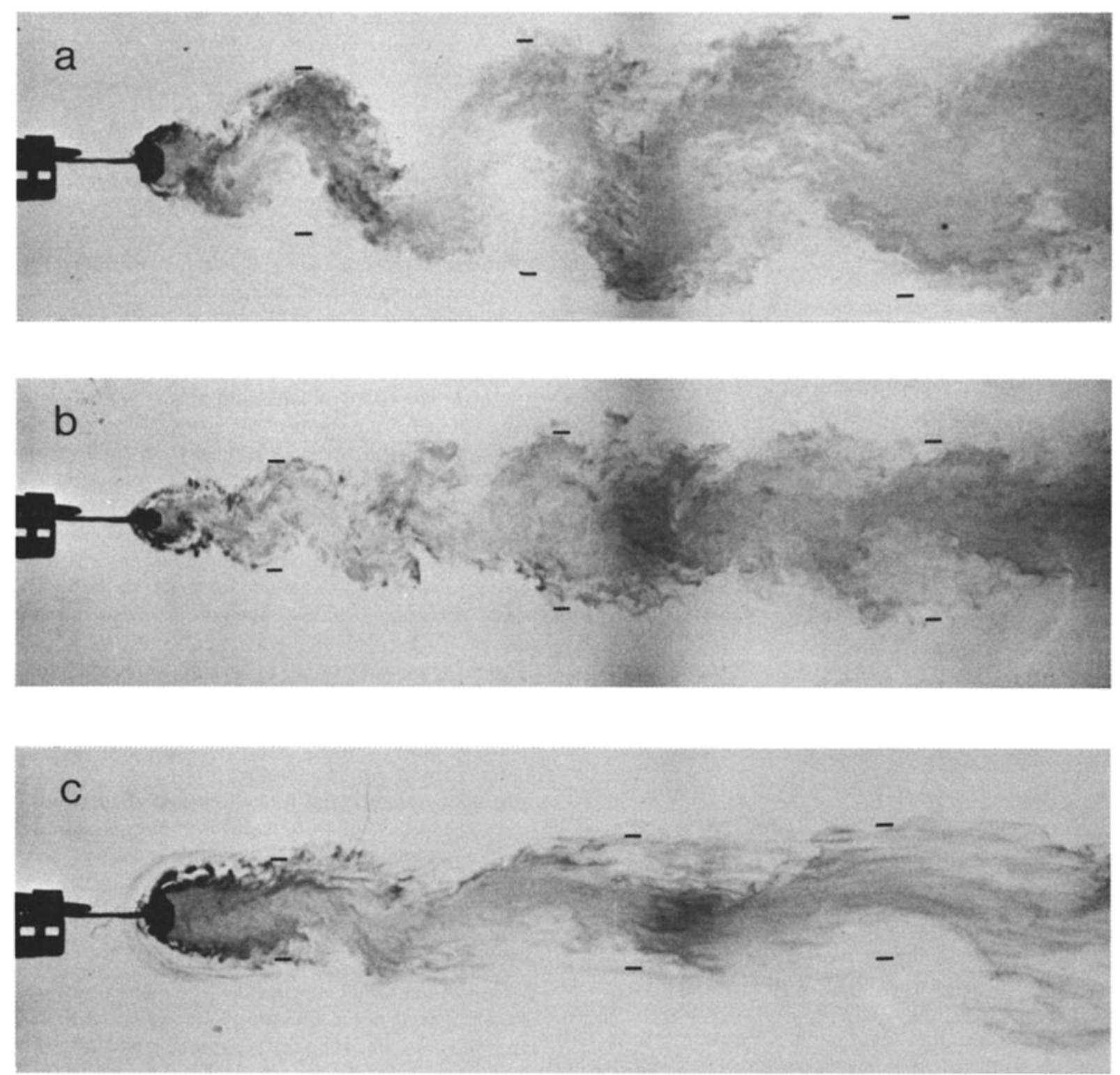

Fig. 5. Wakes on a shallow water table: (a) test A2, (b) test A3, and (c) test B2.

Figure 5 shows the wake structure. The distance between markings in the photographs was taken to be the visual wake width $b$. In Figure 6, one sixth of this visual width, $b / 6$, was compared with the half width $\delta$ predicted by integral analysis. (According to the work of Townsend [1956], in the twodimensional wake of a circular cylinder the turbulent intermittency factor is about $5 \%$ at a lateral position $y=3 \delta$. If the location where the turbulent intermittency factor equals $5 \%$ is taken as the visual boundary, $b$ would be equal to $6 \delta$.) The data in Figure 6 follow reasonably well the line obtained from (12) and (16) by taking a critical value $S_{c}=0.165$.

\subsection{Asymptotic Wake Width}

As the wake approaches the asymptotic width, the entrainment coefficient reduces to zero (equation (16)). For tests A2 and A3 this approach toward asymptotic width occurs at $x c_{f} / h \simeq 0.5$. At this position the momentum defect $M \simeq$ $0.6 M_{0}$. The wake is far from being nullified. However, the growth of the wake is arrested, because the bottom friction stability parameter exceeded the critical value of about 0.165 .
Figure 7 summarizes the data for the asymptotic width and compares them with the prediction by integral analysis. The data obtained from series A, i.e., A1, A2, and A3, are in good agreement with prediction. The Reynolds number for the $B$ series of tests was low $(\operatorname{Re}=4 h / v=4680)$. The results of test series $B$ were affected to some degree by viscosity. In these tests a wake bubble forms downstream of the obstacle but is unable to sustain the oscillation that is necessary for the formation of a trailing vortex (see Figure $5 c$ ). Results of test series B were included to demonstrate how the oscillation in the wake bubble can be suppressed, although the suppression of wake oscillation in this case may be dependent on the stabilizing influence of both viscosity and bottom friction. In the field the oscillation of the wake bubble does not occur until $D c_{f} / h$ approaches a value of about unity.

\subsection{Aspect Ratio of the Vortex Street Wake}

The wavelength $\lambda$ of the vortex street was also obtained from photographs. The vortex street was quite well defined for test $\mathrm{A} 2$, as shown in Figure $5 a$. The aspect ratios $b / \lambda$ for the 


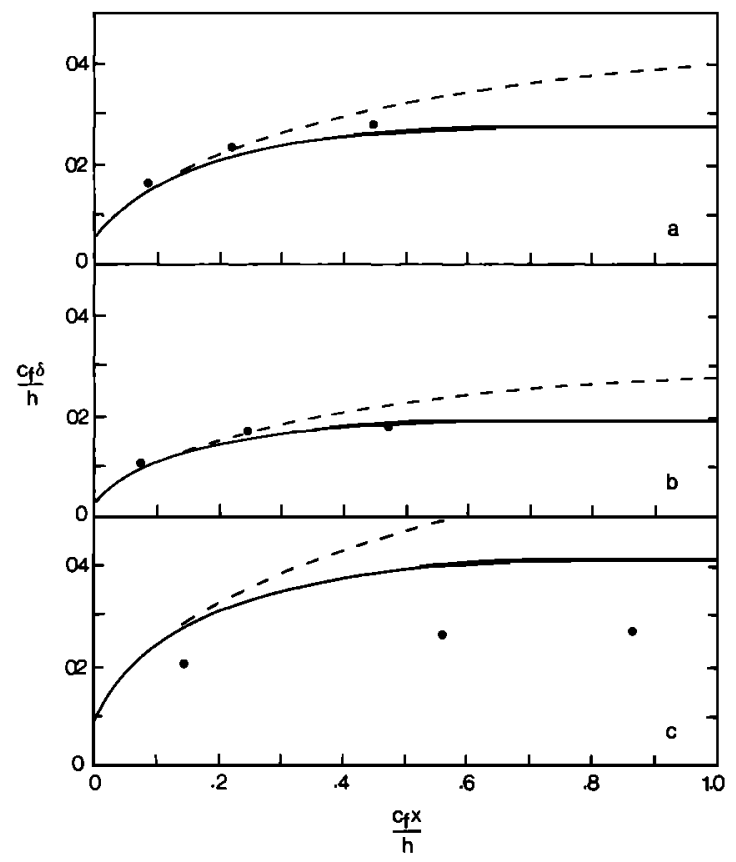

Fig. 6. Prediction of wake widths compared with laboratory observation: (a) test $\mathrm{A} 2,(b)$ test $\mathrm{A} 3$, and (c) test B2. In this figure, $\alpha_{0}=0.09$; solid line, $S_{c}=0.165$; dashed line, $S_{c} \rightarrow \infty$.

three waves in this test were $0.88,0.96$, and 0.79 , with an average value of $b / \lambda \simeq 0.88$. The vortex streets for the other tests in series A were not so well defined but appear to have approximately the same aspect ratio. The vortex streets for test series B were less discernible but appear to have a significantly smaller aspect ratio, with $b / \lambda$ of about 0.36 .

\subsection{Wake Stability Parameter}

The mechanism for suppression of a wake oscillation by bottom friction can be explained by an order-of-magnitude argument. The oscillation is the result of active interaction between the shear layers developed along the two sides of the wake bubble. The oscillation of the wake bubble is suppressed if the shear layers are stabilized by bottom friction. At the end of the bubble the width of the shear layer is $\simeq D$, the mean convection velocity $\bar{U} \simeq U_{1} / 2$, and the transverse shear $\bar{U}_{y} \simeq$

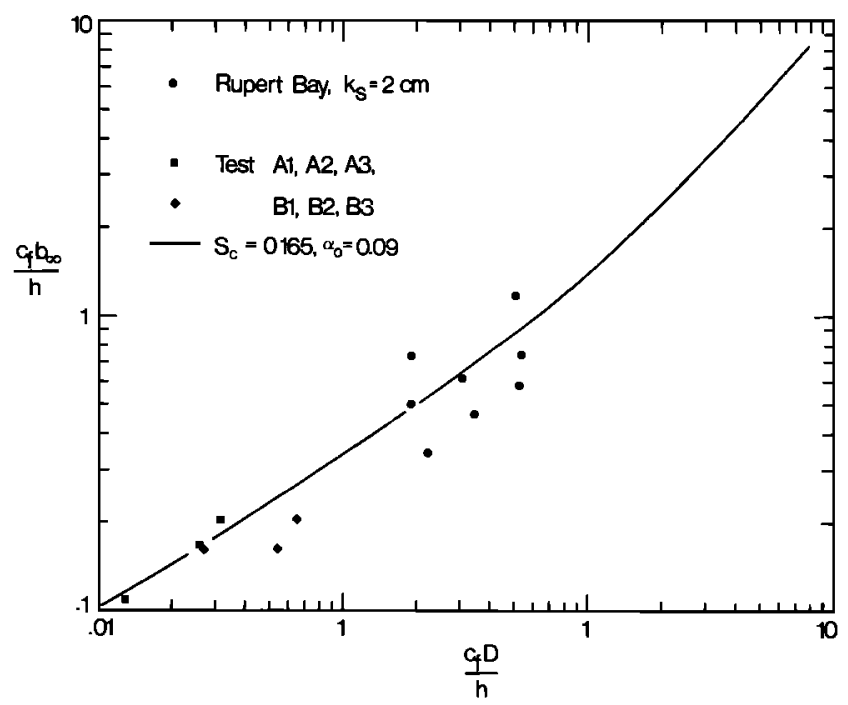

Fig. 7. Asymptotic wake width, $c_{f} b_{o} / h$.
$U_{1} / D$. Thus the bottom friction parameter is given by

$$
S=c_{f} \bar{U} / 2 h \bar{U}_{y}=\frac{1}{4} c_{f} D / h=\frac{1}{4} S_{w}
$$

If the critical $S$ value for the free shear layer is taken as 0.120 (the same value as obtained by Chu et al. [1983] for a hyperbolic tangent velocity profile), the critical value for the wake stability parameter $S_{w}$ would be 0.48 . Thus a vortex street wake is expected if the wake stability parameter is less than the critical value of 0.48 . On the other hand, if the wake stability parameter is sufficiently large, the shear layer developed along the two sides of the island may be stabilized by bottom friction and, in that case, a clear water wake bubble may be observed.

The value of $S_{w}$ for the six tests listed in Table 1 varies from 0.013 to 0.065 , which is an order of magnitude smaller than the critical value obtained above from an order-of-magnitude argument. The kind of clear water wake observed in Rupert Bay is difficult to simulate in the laboratory. The laboratory data were included in this paper primarily for calibration of the integral model.

\section{Island WaKes in Rupert Bay}

We have seen how bottom friction limits the growth of the wake on a shallow water table. The flows around the islands of Rupert Bay are subject to a much stronger influence of bottom friction. A total of 27 different flow configurations around islands in the bay were examined. The cross-stream diameters of the islands vary from 40 to $8700 \mathrm{~m}$, while the offshore depth near the islands ranges from 1 to $5 \mathrm{~m}$. Table 2 summarizes the flow conditions around the islands used in this study. Figure 2 shows the location of the islands mentioned herein and the general bathymetry in the region. There is

TABLE 2. Summary of Flow Conditions Around Islands of Rupert Bay

\begin{tabular}{|c|c|c|c|c|c|c|c|c|c|c|}
\hline Event & Island & $\begin{array}{l}D \\
\mathrm{~m}\end{array}$ & $\begin{array}{l}h, \\
\mathrm{~m}\end{array}$ & $\begin{array}{l}U_{1}, \\
\mathrm{~m} / \mathrm{s}\end{array}$ & $c_{f}^{*}$ & $\begin{array}{c}4 h U_{1} / \nu \\
\times 10^{-6}\end{array}$ & $\begin{array}{c}b_{\infty} \\
\mathbf{m}\end{array}$ & $\lambda$ & $\begin{array}{c}h / U^{3} \\
\mathbf{m}^{-2} \mathbf{s}^{3}\end{array}$ & $S_{w}$ \\
\hline 1 & 1 & 100 & 3 & 0.70 & 0.0056 & 8.4 & 400 & 560 & 8.7 & 0.19 \\
\hline 2 & 1 & 100 & 3 & 0.70 & 0.0056 & 8.4 & 270 & 460 & 8.7 & 0.19 \\
\hline 3 & 2 & 40 & 1 & 0.70 & 0.0076 & 2.8 & 80 & 250 & 2.9 & 0.30 \\
\hline 4 & 3 & 280 & 3 & 0.30 & 0.0056 & 3.6 & 310 & 1050 & 111 & 0.52 \\
\hline 5 & 3 & 280 & 3 & 0.10 & 0.0056 & 1.2 & 390 & 1050 & 3000 & 0.52 \\
\hline 6 & 4 & 160 & 2 & 0.10 & 0.0063 & 0.8 & 375 & 1000 & 2000 & 0.50 \\
\hline 7 & 4 & 160 & 2 & 0.30 & 0.0062 & 2.4 & 375 & 730 & 74 & 0.50 \\
\hline 8 & 5 & 110 & 2 & 0.40 & 0.0062 & 3.2 & 150 & 375 & 31 & 0.34 \\
\hline 9 & 6 & 70 & 2 & 0.40 & 0.0062 & 3.2 & 110 & 270 & 31 & 0.22 \\
\hline 10 & 7 & 3460 & 2 & 0.70 & 0.0062 & 5.6 & no e & ddies & 5.8 & 10.7 \\
\hline 11 & 8 & 560 & 2 & 0.70 & 0.0062 & 5.6 & no e & ddies & 5.8 & 1.74 \\
\hline 12 & 9 & 1310 & 2 & 0.70 & 0.0062 & 5.6 & no ed & ddies & 5.8 & 4.07 \\
\hline 13 & 10 & 1160 & 1 & 0.70 & 0.0076 & 2.8 & no ex & ddies & 2.9 & 8.80 \\
\hline 14 & 10 & 1160 & 1 & 0.60 & 0.0076 & 2.4 & no e & ddies & 4.6 & 8.80 \\
\hline 15 & 9 & 1310 & 2 & 0.60 & 0.0062 & 4.8 & no ed & ddies & 9.3 & 4.07 \\
\hline 16 & 11 & 8700 & 2 & 0.80 & 0.0062 & 6.4 & no ed & ddies & 3.9 & 27.0 \\
\hline 17 & 12 & 1150 & 1 & 0.40 & 0.0076 & 1.6 & no ec & ddies & 16 & 15.6 \\
\hline 18 & 1 & 40 & 4 & 0.30 & 0.0052 & 4.8 & no ed & ddies & 148 & 0.05 \\
\hline 19 & 9 & 1310 & 4 & 0.30 & 0.0052 & 4.8 & no ec & ddies & 148 & 1.70 \\
\hline 20 & 10 & 1160 & 3 & 0.30 & 0.0056 & 3.6 & no ec & ddies & 111 & 2.16 \\
\hline 21 & 13 & 400 & 4 & 0.40 & 0.0052 & 6.4 & no ec & ddies & 63 & 0.52 \\
\hline 22 & 14 & 960 & 3 & 0.40 & 0.0059 & 4.8 & no ec & ddies & 47 & 1.79 \\
\hline 23 & 15 & 130 & 5 & 0.40 & 0.0049 & 8.0 & no ec & ddies & 78 & 0.13 \\
\hline 24 & 16 & 700 & 5 & 0.40 & 0.0049 & 8.0 & no ed & Idies & 78 & 0.69 \\
\hline 25 & 4 & 160 & 4 & 0.40 & 0.0052 & 6.4 & no ec & Adies & 63 & 0.21 \\
\hline 26 & 8 & 560 & 4 & 0.30 & 0.0052 & 4.8 & no ed & Idies & 148 & 0.73 \\
\hline 27 & 1 & 70 & 4 & 0.30 & 0.0052 & 4.8 & no ed & Idies & 148 & 0.09 \\
\hline
\end{tabular}

*The value of $k_{\mathrm{s}}$ is $2 \mathrm{~cm}$. 
some uncertainty in the offshore depth estimation for many of the islands because of a sparcity of depth information in the shallow areas outside of the main channels in this isolated region of northern Canada. Depths for "lowest normal tides" were obtained from a Canadian Hydrographic Service [1972] chart which was constructed from a 1912-1913 survey. Depths were interpolated and extrapolated from the bathymetric contours with adjustment for tide height fluctuations relative to the chart bench mark. A further degree of uncertainty occurs because this area is one of rapid postglacial uplift. For this reason, depths listed in Table 2 are typically accurate within $\pm 1 \mathrm{~m}$. Island diameter $D$ was determined by measuring the width of the land area perpendicular to the incident flow in the aerial photographs. Incident velocity $U_{1}$ was estimated from current meter values taken at a depth of $2.5 \mathrm{~m}$ at seven locations in the bay. Because of the horizontal separation between the individual island and the mooring sites, $U_{1}$ values are assumed to be accurate within $\pm 0.10 \mathrm{~m} / \mathrm{s}$. The estimated values compared favorably with those found by Ouellet [1977] in his numerical model of flow in Rupert Bay. It should be noted that the same island may have different $D$ and $h$ values because of varying tidal height and current orientation.

The bottom friction coefficient in Table 2 was calculated by the Colebrook-White formula for pipe flow. The equivalent Nikuradse's sand grain roughness $k_{s}$ was assumed to be equal to $2 \mathrm{~cm}$. The relative roughness is $k_{s} / 4 h$. The value of $c_{f}$, in the range of 0.0049 to 0.0076 , is higher than those obtained by Sternberg [1968] from the six tidal channels in Puget Sound. The high friction coefficient comes from the shallow depth of Rupert Bay. In such a shallow area the roughness is enhanced by wind-wave induced oscillation at the bottom [Grant and Madsen, 1986]. The 2-cm sand grain roughness was chosen largely to fit the field observations. However, the friction coefficient obtained follows the trend of data shown by Sternberg [1968]. Ouellet [1977] found a Chezy coefficient of $C=40$ worked best for his numerical model of tidal flow in Rupert Bay. The value of the Chezy coefficient $\left(C=\left(2 g / c_{f}\right)^{1 / 2}\right)$ in Table 2 is in the range of 63 to 51 , or slightly higher.

\subsection{Vortex Street Wakes}

Vortex streets were observed in the island wakes on nine occasions. Figure 3 shows an aerial view of some of the vortex streets. Flow conditions, the width of the wake, $b_{\infty}$, and the wavelength between wave crests, $\lambda$, are listed in Table 2 . The aspect ratios are

$$
b_{\infty} / \lambda=0.71,0.59,0.32,0.30,0.37,0.38,0.51,0.40,0.41
$$

for the events $1-9$, respectively. These values are comparable in order of magnitude with the laboratory observations, where $b_{\infty} / \lambda \simeq 0.9$ for test $\mathrm{A} 2$, while $b_{\infty} / \lambda \simeq 0.36$ for the nearly collapsed wake, test $B 2$.

According to the stability analysis of parallel flow, given in the appendix, the most unstable wave has a wave number

$$
k=2 \pi \delta / \lambda \simeq 0.8
$$

This leads to a value of $\delta / \lambda \simeq 0.13$, or an aspect ratio $b / \lambda=0.78$ if the visual width $b$ is equal to $6 \delta$.

The wake stability parameter, $S_{w}=c_{f} D / h$, for the nine events ranges from 0.19 to 0.52 (Table 2). These values are one order of magnitude larger than the laboratory values listed in Table 1 . The vortex wake in Rupert Bay is clearly much more stable than those observed in the laboratory.
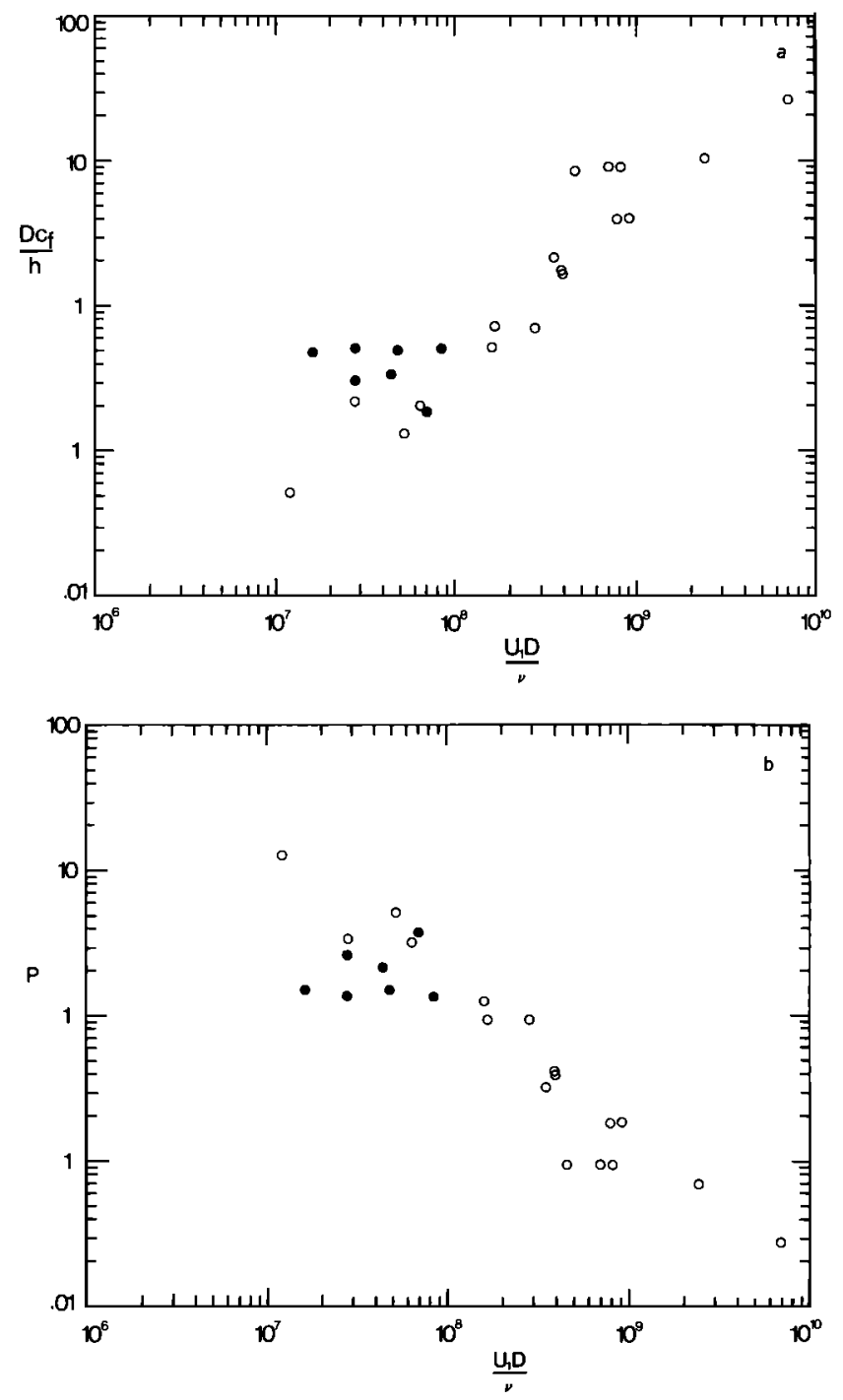

Fig. 8. Flow conditions of the 27 wake events in Rupert Bay. Solid circles identify the vortex street wake. (a) Classification by $S_{w}$. (b) Classification by $P$.

A comparison of the asymptotic wake width $c_{f} b_{\infty} / h$ with the integral analysis is given in Figure 7. The solid line is the asymptotic wake width, $6 \delta_{\infty} c_{f} / h$, obtained from (12) and (16) with $S_{c}=0.165$ and $\alpha_{0}=0.09$.

\subsection{Stability of the Wake Bubble: Classification}

Vortex wakes were not observed for the remaining 18 events. The question of whether a vortex street forms depends on the value of the wake stability parameter $S_{w}$, as discussed in section 3.3.

Figure $8 a$ shows the values of the wake stability parameter for the 27 cases found in Rupert Bay. Vortex street wakes were observed over a range of $S_{w}$ varying from 0.19 to 0.52 . This is consistent with the order-of-magnitude argument in section 3.3, where a critical $S_{w}$ value of 0.48 was obtained. For a wake stability parameter greater than about 0.5 the shear layer is stabilized by bottom friction, and generation of a vortex street wake is impossible.

However, there are four events $(18,23,25$, and 27$)$ which do not follow the rule. The wake stability parameters for these four events were $0.05,0.13,0.21$, and 0.09 , respectively. Al- 
though they were below the critical value of about 0.5 , vortex streets were not observed.

To understand the reason for this discrepancy, one must consider the hydrodynamic conditions at the time of the observations. Aerial photographs were obtained over a $2-3 \mathrm{~h}$ period on two different days. On one occasion this corresponded to a tidal phase range from $4 \mathrm{~h}$ after high tide $(H+4)$ to low tide $(L)$, while the second flight took place from just after $H$ to $H+2$. The four events which do not fit the $S_{w}$ dependence occurred in the second series. During the period from $H$ to $H+3$ the water column is weakly stratified with a pycnocline depth of about $2 \mathrm{~m}$ in the area downstream of Stag Rock (island 3). Salinity differences across the pycnocline over this interval are typically $1-2 \mathrm{~g} / \mathrm{kg}$. For the remainder of the tidal cycle the water column approximates vertical homogeneity. Thus the four low- $S_{w}$ events for which no vortex street was generated occurred during a period of water column stratification. Events 8 and 9, with vortex streets, also occurred during this period but in areas with much smaller depths. The offshore area near these two islands was above water at low tide. In looking for another criterion which may be applied at the same time as the $S_{w}$ dependence to determine if a vortex street will be generated, we consider the $h / U^{3}$ relationship formulated by Simpson and Hunter [1974]. Parameter values of order 60 (mks units) and greater signify a stratified water column. Given the imprecision in the depth and velocity estimates, one finds the four nonvortex street events occurred at times of a stratified water column, as determined by the Simpson-Hunter parameter (Table 2). Stratification will both limit the upward resuspension of bottom sediments, thus making vortex visualization by aircraft diflicult, and modify the frictional effects by introduction of a pycnocline. In examining the cases when a vortex street was generated in spite of a high $h / U^{3}$ value, one finds four events $(4,5,6$, and 7$)$. At the time of these events $(H+5$ to $L)$ the water column was completely fresh at each location, so the stratification parameter value is not appropriate. These events can be considered to have occurred for vertically well-mixed conditions, similar to the others observed.

An alternate method of classification according to Wolanski et al. [1984a] is given in Figure $8 b$. They proposed the use of the parameter

$$
\begin{gathered}
P=U_{1} h^{2} / v_{T} D \\
\text { in which } v_{T}=0.15 h U_{*}=0.15 h U_{1}\left(c_{f} / 2\right)^{1 / 2} \text {. Thus } \\
P=\left[\left(2 c_{f}\right)^{1 / 2} / 0.15\right] S_{w}{ }^{-1}
\end{gathered}
$$

Since the variation of $c_{f}$ is not large, $P$ is directly related to $S_{w}$. Thus the two methods of classification, by $S_{w}$ or by $P$, are practically the same. The values of $P$ for the nine events with vortex streets range from 1.3 to 3 . Again, there are four events with a $P$ value greater than 1.3 but without the sighting of a vortex street. This discrepancy can be accounted for using similar aguments to those of the $S_{w}$ dependence.

\subsection{Sheltering Effect of Large Islands}

For flow around large islands the transverse shear is very weak. In this case the shear layers are practically laminar and are strongly affected by the stable influence of bottom friction. Typical flows around large islands are shown in Figures $3 e$ and $3 f$. A clear water wake of limited extent with low turbulence and low sediment suspension is observed on the lee side of the island. The wake stability parameter, $S_{w}$, was large in both cases.

A qualitative simulation of the flow within the recirculating bubble can be made by a simple finite difference calculation. For simplicity the calculations were made for flow around a rectangular island. Figure 9 shows the typical streamline pattern for three values of wake stability parameter. The Reynolds number for all cases is $D U_{1} / v_{T}=300$.

The calculation was made using the stream function and vorticity formulation technique as described by Peyret and Taylor [1983]. A $20 \times 80$ grid was used to cover one half of the flow field. The width of the obstacle shown in the figure is $D / 2$. For the values of $S_{w}=0.012,0.12$, and 1.2 the length of the eddy is $L / D=4.39,2.74$, and 0.601 , respectively. The reduction of circulation in the wake bubble is quite noticeable as the wake bubble is stabilized by the influence of bottom friction. For large $S_{w}$ the flow in the wake bubble is sheltered by the island to form a zone of "dead water" with low turbulent activity and suspended sediment concentration.

The stabilizing influence of bottom friction is different from the stabilization influence of viscosity. The effect of changing viscosity is demonstrated in Figure 10. While the length of the eddy depends on the change in Reynolds number, the flow recirculation within the wake bubble is relatively unaffected.

It should be pointed out that this numerical simulation, with uniform eddy viscosity throughout the flow field, is not quite realistic. Nevertheless, it demonstrates how a dead water area of very low circulation velocity can be created not by the stable influence of viscosity but by bottom friction.

\section{Conclusions}

Rupert Bay is an unusual natural setting for visualization of transverse shear flows in shallow depth. By analyzing the
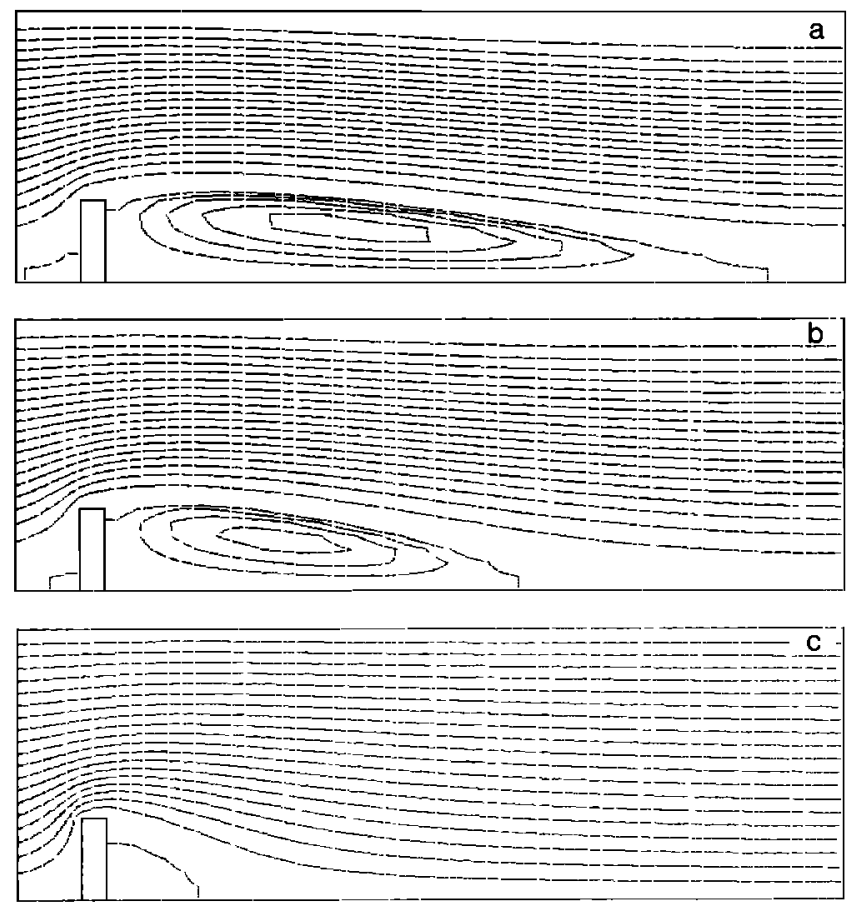

Fig. 9. Flow separation behind an obstacle in shallow water; Reynolds number is $D U_{1} / v_{T}=300$; flow rates between streamlines are $\Delta \psi=0.05$ and 0.005 , respectively, for flows inside and outside of the wake bubble. (a) $S_{w}=0.012,(b) S_{w}=0.12$, and (c) $S_{w}=1.2$. 

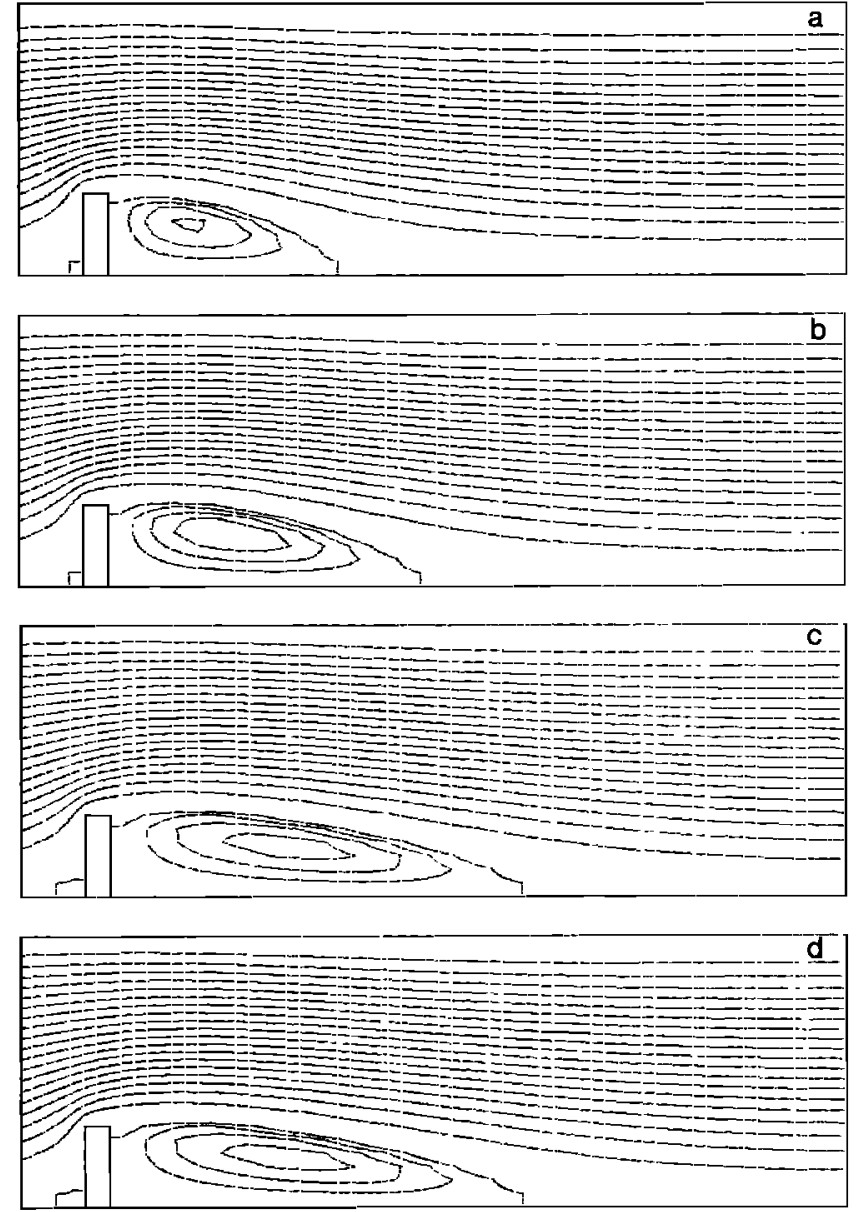

Fig. 10. Flow separation behind an obstacle in shallow water; wake stability parameter $S_{\mathrm{w}}=c_{f} D / h=0.12 ;$ (a) $D U_{1} / v_{T}=60$, (b) $D u_{1} / v_{T}=120$, (c) $D U_{1} / v_{T}=300$, and (d) $D U_{1} / v_{T}=600$. Flow rates between stream lines are $\Delta \psi=0.05$ and 0.005 , respectively, for flow inside and outside the wake bubble.

aerial photographs of island wakes in Rupert Bay and comparing them with laboratory observations and an analytical description of wakes in shallow water, we have been able to piece together a consistent picture on the scale of transverse shear flow and how it is limited by the effect of bottom friction.

Similar transverse shear flows, with a large horizontal length scale compared to depth, are observed in the Earth's atmosphere and oceans. The length scales of these shear flows may be very different from those in Rupert Bay. Some may be affected by buoyancy, Coriolis effects, and other dynamic processes. But a similar stabilizing effect on the turbulent motion is anticipated.

The presence of this stabilizing effect of bottom friction, or of interfacial friction in stratified flows, makes the energycascading process of the large-scale transverse shear flow, in the Earth's atmosphere and oceans, very different from the conventional turbulent flow problem. Transverse motion, with a length scale large compared with $h / c_{f}$, cascades its energy directly toward the very small scale through bottom friction. As a result, there is a lack of motion in a range of length scale from the mean flow to the scale $h / c_{f}$ ( $h$ being the depth of the flow and $c_{f}$ the friction coefficient). Perhaps this is also the reason for the laminar appearance of atmospheric flow and oceanic motions commonly observed using aerial photography.

\section{APPENDix: Stability Analysis}

Chu et al. [1983] analyzed the stability of parallel shear flows with hyperbolic tangent and hyperbolic secant velocity profiles and found critical values of the bottom friction stability parameter to be 0.120 and 0.145 , respectively. A similar analysis is carried out here for the Gaussian velocity profile, so as to simulate the wake flow. The Gaussian profile is

$$
\tilde{U}=\widetilde{U}_{1}+\exp \left[-(\ln 2) \tilde{y}^{2}\right]
$$

in which $\tilde{U}, \tilde{U}_{1}$, and $\tilde{y}$ are dimensionless variables, normalized by the velocity scale $u_{m}$ and the half width $\delta$. Following the classical approach, one can introduce a small disturbance into the mean flow. The condition for neutral stability is determined by numerical methods as described by Chu et al. [1983]. In general, the stability of the parallel flow depends on the wave number

$$
k=2 \pi \delta / \lambda
$$

and the bottom friction stability parameter

$$
S=c_{f} U / 2 h U_{y}
$$

in which $c_{f}, U$, and $U_{y}$ are, respectively, the friction coefficient, the velocity, and the velocity gradient evaluated at the inflexion point of the Gaussian profile. There are two modes of oscillations for the Gaussian velocity profile. The sinuous mode is most unstable. Figure A1 shows the neutral stability curves for the sinuous mode. The most unstable sinuous waves are those with a wave number $k \simeq 0.8$. The flow is stable when the bottom friction parameter exceeds the critical value $S_{c}$ of about $0.16 \sim 0.17$.

It should be pointed out that this result, presented in Figure A1, is for parallel and laminar flow. A similar influence of bottom friction on the island wake is anticipated, although the flow around the island is turbulent and not quite parallel.

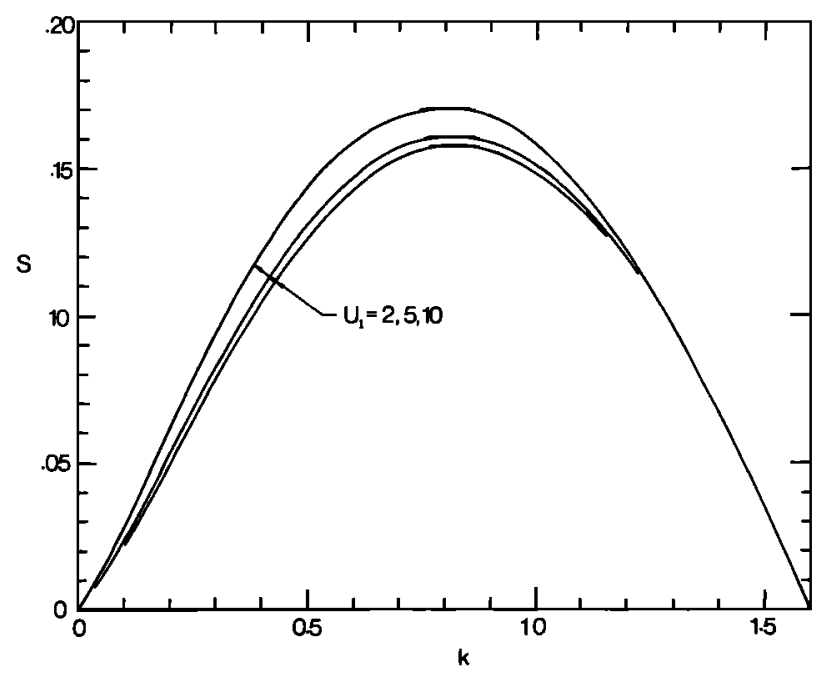

Fig. A1. Neutral stability curves for a Gaussian velocity profile. 
Acknowledgments. This work was supported by research grants from Natural Sciences and Engineering Research Council of Canada to both authors. Fieldwork was made possible by funding from the Societe d'Energie de la Baie James and Hydro-Quebec. We acknowledge the helpful comments of Paul Leblond. Aerial photographs (copyright year 1976) in this article were reproduced from the collection of the National Air Photo Library with permission of Energy, Mines and Resources Canada. Contribution to the program Groupe Interuniversitaire de Recherches Oceanographiques du Quebec.

\section{REFERENCES}

Babarutsi, S., and V. H. Chu, Experimental study of turbulent mixing layers in shallow open channel flows, Tech. Rep. 85-1, Fluid Mech. Lab., Dep. of Civ. Eng. and Appl. Mech., McGill Univ., Montreal, Que., Canada, 1985.

Batchelor, G. K., An Introduction to Fluid Dynamics, 615 pp., Cambridge University Press, New York, 1967.

Burger, E., and R. Wille, Periodic flow phenomena, Annu. Rev. Fluid Mech., 4, 313-340, 1972.

Canadian Hydrographic Service, Chart 5414, scale 1:73,000, Ottawa, 1972.

Chopra, K. P., and L. F. Hubert, Mesoscale eddies in the wake of islands, J. Atmos. Sci., 22, 652-657, 1965.

Chow, V. T., Open-Channel Hydraulics, p. 10, McGraw-Hill, New York, 1959.

Chu, V. H., and M. T. H. Abdelwahed, Turbulent wakes in shallow waters, Publ. $81-$ FE-13, 7 pp., Am. Soc. of Mech. Eng., New York, 1981.

Chu, V. H., J.-H. Wu, and R. E. Khayat, Stability of turbulent shear flows in shallow channels, in Proceedings of the 20th IAHR Con- gress, Moscow, vol. 3, pp. 128-133, International Association for Hydraulic Research, 1983.

Grant, W. D., and $O$. S. Madsen, The continental-shelf bottom boundary layer, Annu. Rev. Fluid Mech., 18, 265-305, 1986.

Ouellet, Y., Etude à l'aide d'un modele mathématique de la circulation hydrodynamique, de la propagation de la marée et de la distribution de la salinité dans la baie de Rupert, tech. rep., 24 pp., Soc. D'Energ. de la Baie James, Montreal, Quebec, Canada, 1977.

Peyret, R., and T. D. Taylor, Computational Methods for Fluid Flow, 358 pp., Springer-Verlag, New York, 1983.

Simpson, J. H., and J. R. Hunter, Fronts in the Irish Sea, Nature, 250, $404-406,1974$.

Sternberg, R. W., Friction factors in tidal channels with differing bed roughness, Mar. Geol., 6, 243-260, 1968.

Townsend, A. A., The Structure of Turbulent Shear Flows, Cambridge University Press, New York, 1956.

Wolanski, E., G. L. Pickard, and D. L. B. Jupp, River plumes, coral reefs and mixing in the Gulf of Papua and Northern Great Barrier Reef, Estuarine Coastal Shelf Sci., I8, 291-314, 1984a.

Wolanski, E., J. Imberger, and M. L. Heron, Island wakes in shallow coastal waters, J. Geophys. Res., 89(C6), 10,553-10,569, $1984 b$.

V. H. Chu, Department of Civil Engineering, McGill University, 817 Sherbrooke Street West, Montreal, Quebec, Canada H3A 2K6.

R. G. Ingram, Department of Meteorology, McGill University, 805 Sherbrooke Street West, Montreal, Quebec, Canada H3A 2K6.

(Received April 20, 1987; accepted June 15, 1987.) 